\title{
The effect of zoledronate during bone healing
}

\author{
Marcos Almeida Matos • Uenis Tannuri • \\ Roberto Guarniero
}

Received: 1 October 2009/Accepted: 27 January 2010/Published online: 19 February 2010

(C) Springer-Verlag 2010

\begin{abstract}
Background Bisphosphonates have become the treatment of choice for a variety of bone diseases in which excessive osteoclastic activity is an important pathologic feature. However, inhibition of osteoclastic activity could lead to inhibition of remodeling during bone healing or repair. The objective of this study is to investigate the effect of zoledronate (the most potent bisphosphonate) in the biological process of bone healing.

Methods Thirty immature male rabbits were divided into two groups (control and experimental) of 15 animals each. Both groups were submitted to fibular osteotomy. Only in the experimental group a single dose of zoledronate was administered. After 1, 2, and 4 weeks, animals of both groups were euthanized and the osteotomy site was histomorphometrically evaluated. The associated parameters analyzed were tissue volume (TV), fractional trabecular bone volume (BV/TV), fractional woven bone volume (WoV/TV), fractional periosteal fibrous volume (FbV/TV), and medullary fibrous volume (MaV/TV).

Results The first week of healing was characterized by small callus area (experimental group) and less periosteal

M. A. Matos ( $\square)$

Department of Orthopedics and Traumatology,

Bahian School of Medicine and Public Health, Rua da Ilha,

378, casa 21, Itapuã, Salvador-Bahia 41620-620, Brazil

e-mail: malmeidamatos@ig.com.br

U. Tannuri

Department of Surgery,

São Paulo University, São Paulo, Brazil

R. Guarniero

Department of Orthopedics and Traumatology,

São Paulo University, São Paulo, Brazil
\end{abstract}

fibrosis. The second week was characterized by a large quantity of woven bone and marked decrease in periosteal fibrosis in the two groups. In the control group there was also a significant increase in trabecular bone. The fourth week was characterized by increased amount of woven bone and trabecular bone in the experimental group; there was increased medullary fibrosis in the two groups, while there continued to be significantly less periosteal fibrosis in the experimental group.

Conclusions Zoledronate does not prevent bone healing. However, the effect of zoledronate was characterized by accentuated stimulation of primary bone production and probably inhibition of remodeling, leading to retention of trabecular bone.

Keywords Zoledronate · Bone healing ·

Bisphosphonate $\cdot$ Experimental

\section{Introduction}

Bisphosphonates (BS) can be classified into two major groups. The first group comprises the non-nitrogen-containing BS and the second group contains the more potent, nitrogen-containing $\mathrm{BS}$ such as alendronate, pamidronate, risedronate, and zoledronate (ZA). Members of the second group interfere with the mevalonate biosynthetic pathway and affect cellular activity and cell survival by interfering with protein prenylation [1]. Prenylation is required for important signaling proteins that regulate a variety of cell processes important to osteoclast function, including cell morphology, cytoskeletal arrangement, membrane ruffling, trafficking of vesicles, and apoptosis [2]. Bone resorption is mediated by osteoclasts and its activity can be reduced by systemic BS treatment. 
The most impressive clinical application of BS has been as inhibitors of bone resorption, especially for diseases for which no effective treatment existed previously. Thus, BS have become the treatment of choice for a variety of bone diseases in which excessive osteoclastic activity is an important pathologic feature, including Paget's disease of bone, metastatic and osteolytic bone disease, hypercalcemia of malignancy, osteogenesis imperfecta, as well as osteoporosis [3].

In spite of BS treatment, pathologic fractures and the need for surgical orthopedic treatments that require bone remodeling are common in such diseases. Inhibition of osteoclastic activity could lead to inhibition of remodeling during bone healing or repair or bone graft incorporation. As inhibitors of remodeling, the effects of BS during these processes remain unknown and could constitute a negative influence [1-3].

The effects of BS on fracture healing have been investigated after administration of clodronate, alendronate, etidronate, and incadronate [4-6]. Most of the previous studies did not report significant changes during fracture repair, while others showed slight improvement in the amount of bone formed. Kiely et al. [7] showed increase in new bone formation when zoledronate was used in a distraction osteogenesis model and suggested that BS could have a positive effect for that drug. Zoledronate also improves the amount of bone in the metaphysis during bone healing, suggesting a positive effect on remodeling [8].

Zoledronate is the most potent BS in clinical use and its effects on remodeling could demonstrate the role of this group of drugs during repair or bone healing process more than any other BS. The objective of this study is to check the effect of zoledronate in the biological process of bone healing, in a controlled experimental model in rabbits submitted to fibular osteotomy in accordance with the method described by Matos et al. [9, 10].

\section{Materials and methods}

This study conformed to the guiding principles of the Declaration of Helsinki involving experimental animals and was approved by the Research Ethics Committees at the University of São Paulo and at the Bahia School of Medicine and Public Health.

Animals: experimental groups

Thirty immature male albino New Zealand rabbits were divided into two groups of 15 animals each, assigned to the control and experimental group, respectively. The animals were 6-8 weeks old when the experiment began in both groups. Initial weight was $918 \mathrm{~g}$ in the control group and $875 \mathrm{~g}$ in the experiment group (no significant difference between groups by $t$-test).
The animals were acclimatized in the animal care facility for several days and were housed in individual cages during the entire study period with water and pellet chow diet ad libitum.

\section{Experimental design}

Food was suspended $8-10 \mathrm{~h}$ prior to administering anesthesia. To decrease the vagal tonus, each animal received $0.2 \mathrm{mg} / \mathrm{kg}$ atropine sulfate by intramuscular injection. Animals were anesthetized by intraperitoneal injection of ketamine (25.0-30.0 mg/kg body weight) and intramuscular injection of diazepam (5.0-10.0 mg/kg body weight).

This experimental osteotomy model in rabbits was reported by Matos et al. [9]. Under aseptic technique conditions, the fibula of each animal was accessed by approximately 5-mm lateral incision on the right pelvic limb. After division of the skin and subcutaneous tissue, the fascia of fibular muscles and periosteum were opened and dissected from the cranial portion of the fibula. Shaft osteotomy was performed on the cranial portion of the exposed fibula, using an electric saw with a standardized blade (10.0 $\mathrm{mm}$ wide, $0.5 \mathrm{~mm}$ thick). The incision was closed in layers, using absorbable 5-0 polyvicryl sutures for the fascia and 5-0 mononylon sutures for the skin.

In the experimental group (group 2), a single dose of $0.04 \mathrm{mg} / \mathrm{kg}$ zoledronate was administered via intraperitoneal injection immediately before the surgical procedure. In the control group (group 1), the same volume of doubledistilled water was administered under similar conditions. Both groups were submitted to the same procedure. After that, groups 1 and 2 were divided into subgroups $\mathrm{A}, \mathrm{B}$, and $\mathrm{C}$, containing five animals each, designated $1 \mathrm{~A}, 1 \mathrm{~B}$, and $1 \mathrm{C}$ for control, and $2 \mathrm{~A}, 2 \mathrm{~B}$, and $2 \mathrm{C}$ for experiment.

After 1 (subgroups A), 2 (for subgroups B), and 4 weeks (subgroups C), animals of both groups were anesthetized and euthanized by intracardiac injection of $2 \mathrm{ml}$ potassium chloride. The fibula of each animal was removed, dissected from the surrounding soft tissue, and fixed in $10 \%$ formalin for microscopic evaluation. Formalin-fixed bones were decalcified with $7.5 \%$ nitric acid, embedded in paraffin, and longitudinally sectioned. Histological sections ( $7 \mu \mathrm{m}$ thick) were stained with hematoxylin and eosin prior to optical microscope examination.

Histomorphometric evaluation of the callus

Three histological sections were analyzed for each animal. After the cuts had been chosen, preliminary analysis was performed at $100 \times$ magnification in order to define the area of the callus, defined by the regions associated with significant periosteal thickening, i.e., the area where the cortical bone thickness had more than doubled. 
Histomorphometric evaluations of all microscopic fields were performed using a test eyepiece reticule with 10 parallel lines and 100 points in a grid with a total area of $10,500 \mu \mathrm{m}^{2}$ (Zeiss 23-9901) at magnification of $200 \times$. The associated parameters analyzed were tissue volume (TV), fractional trabecular bone volume (BV/TV), fractional woven bone volume (WoV/TV), fractional periosteal fibrous volume (FbV/TV), and medullary fibrous volume (MaV/TV), taken according to previous reports by Prafitt et al. [11] and Matos et al. [10].

\section{Statistical analysis}

Difference between mean continuous values was tested using Student's $t$-test (0.05) for parametric data. When verifying hypotheses for nonparametric data comparing more than two distributions, the Kruskal-Wallis test was used, followed by Dunn's post hoc test (when the probability of the former was less than 0.05). In comparison of two independent nonparametric distributions, the MannWhitney test was used at a level of significance of 0.05 .

\section{Results}

\section{Overall assessment}

One animal in the experimental group had slight suture dehiscence but was not withdrawn from the research as it was a superficial lesion that needed no treatment other than a dressing. At the end of the study, all the animals in the two groups were assessed as being in a satisfactorily healthy condition.

\section{Microscopic assessment}

The appearance of the bone callus seen under $40 \times$ magnification confirmed that there were no cases of pseudarthrosis. The microscopic appearance was similar in an overall and qualitative manner. Staining with hematoxylin and eosin allowed good distinction between the bone tissue in primary structure (woven bone), mature bone in trabecular structure, periosteal fibrosis, and medullary fibrosis.

Histomorphometric analysis was performed, quantifying the parameters mentioned in "Materials and methods". Testing of differences in relation to time (in the rows of the tables) was checked by the Kruskal-Wallis test and marked with "\#” (hash) for $P<0.05$ in relation to subgroup 1A; testing of the differences among the control and experimental groups (in the columns of tables) was checked by Mann-Whitney test and marked with “*” (asterisk) for $P<0.05$.
The first week of consolidation was characterized by large callus area (significantly smaller in the experimental group), a large amount of periosteal fibrosis (significantly less in the experimental group), little medullary fibrosis, and little trabecular bone. The second week was characterized by a large quantity of woven bone and marked decrease in periosteal fibrosis in the two groups. In the control group there was also significant increase in trabecular bone. The fourth week was characterized by decrease in callus area in the two groups and reduced amount of woven bone in the control group. The amount of trabecular bone increased significantly in the experimental group. There was increased medullary fibrosis in the two groups, while there continued to be significantly less periosteal fibrosis in the experimental group. The results of this histomorphometric analysis are presented in Figs. 1 and 2 and Tables 1-5.

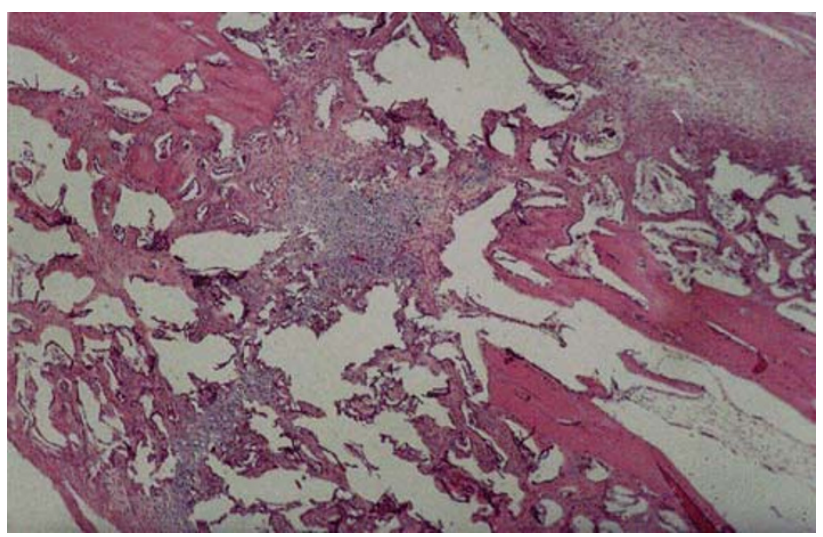

Fig. 1 Histologic section of the callus in the control group during the fourth week of healing (HE, 100×)

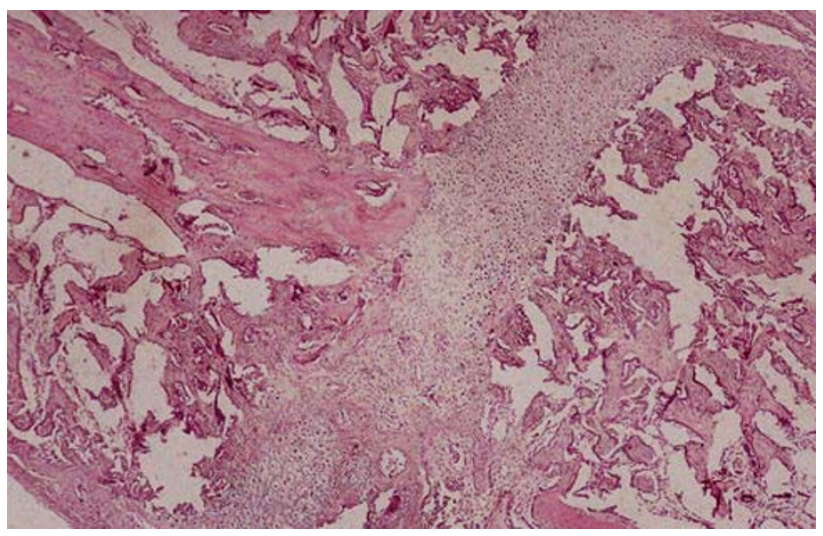

Fig. 2 Histologic section of the callus in the experimental group during the fourth week, showing a large amount of woven bone compared with the control group (HE, 100 $\times$ ) 
Table 1 Tissue volume (TV) $\mathrm{mm}^{3}$

\begin{tabular}{llll}
\hline Group & $\begin{array}{l}\text { First week } \\
\text { Mean (standard deviation) }\end{array}$ & $\begin{array}{l}\text { Second week } \\
\text { Mean (standard deviation) }\end{array}$ & $\begin{array}{l}\text { Fourth week } \\
\text { Mean (standard deviation) }\end{array}$ \\
\hline Control & $314(59)^{* \#}$ & $183(45)$ & $132.4(31)^{\#}$ \\
Experimental & $187.2(10.6)^{* \#}$ & $180.8(8.5)$ & $135.2(24.1)^{\#}$ \\
\hline
\end{tabular}

\# $P<0.05$ for Kruskal-Wallis and Dunn's tests intragroup

* $\quad P<0.05$ for Mann-Whitney test intergroup

Table 2 Fractional woven bone volume (WoV/TV)

\begin{tabular}{lllc}
\hline Group & $\begin{array}{l}\text { First week } \\
\text { Mean (standard deviation) }\end{array}$ & $\begin{array}{l}\text { Second week } \\
\text { Mean (standard deviation) }\end{array}$ & $\begin{array}{l}\text { Fourth week } \\
\text { Mean (standard deviation) }\end{array}$ \\
\hline Control & $18.9(4.2)^{\#}$ & $17.4(6.3)$ & $2.8(2.7)^{* \#}$ \\
Experimental & $23.6(4.6)$ & $23.2(10)$ & $16.1(12)^{*}$ \\
\hline
\end{tabular}

\# $P<0.05$ for Kruskal-Wallis and Dunn's tests intragroup

* $\quad P<0.05$ for Mann-Whitney test intergroup

Table 3 Fractional trabecular bone volume (BV/TV)

\begin{tabular}{lllc}
\hline Group & $\begin{array}{l}\text { First week } \\
\text { Mean (standard deviation) }\end{array}$ & $\begin{array}{l}\text { Second week } \\
\text { Mean (standard deviation) }\end{array}$ & $\begin{array}{l}\text { Fourth week } \\
\text { Mean (standard deviation) }\end{array}$ \\
\hline Control & $7.6(2.8)^{\#}$ & $28.2(3.9)^{\#}$ & $25.9(5.3)$ \\
Experimental & $10(2.3)^{\#}$ & $25.4(7.2)$ & $31(6.8)^{\#}$
\end{tabular}

\# $P<0,05$ for Kruskal-Wallis and Dunn's tests intragroup

* $P<0,05$ for Mann-Whitney test intergroup

Table 4 Fractional medullary fibrous volume (MaV/TV)

\begin{tabular}{llll}
\hline Group & $\begin{array}{l}\text { First week } \\
\text { Mean (standard deviation) }\end{array}$ & $\begin{array}{l}\text { Second week } \\
\text { Mean (standard deviation) }\end{array}$ & $\begin{array}{l}\text { Fourth week } \\
\text { Mean (standard deviation) }\end{array}$ \\
\hline Control & $3.9(2.5)^{\#}$ & $15.8(1.9)$ & $19.2(5.2)^{\#}$ \\
Experimental & $6.3(2.4)^{\#}$ & $21.9(10.5)$ & $29.8(16.4)^{\#}$ \\
\hline
\end{tabular}

\# $P<0.05$ for Kruskal-Wallis and Dunn's tests intragroup

* $\quad P<0.05$ for Mann-Whitney test intergroup

Table 5 Fractional periosteal fibrous volume (FbV/TV)

\begin{tabular}{llll}
\hline Group & First week & Second week \\
& Mean (standard deviation) & Mean (standard deviation) & $\begin{array}{l}\text { Fourth week } \\
\text { Mean (standard deviation) }\end{array}$ \\
\hline Control & $55.2(8)^{* \#}$ & $17.3(4.9)^{* \#}$ & $17.3(4.9)^{* \#}$ \\
Experimental & $42.9(8.7)^{* \#}$ & $8(2.7)^{* \#}$ & $8.4(3.6)^{*}$ \\
\hline
\end{tabular}

\# $P<0.05$ for Kruskal-Wallis and Dunn's tests intragroup

* $P<0.05$ for Mann-Whitney test intergroup

\section{Discussion}

The process of bone and fracture repair can be considered as consisting of anabolic (bone-forming) and catabolic (bone-resorbing) responses. The first step of this process is characterized by endochondral ossification that produces a nonmineralized cartilage scaffold. Osteoblastic cells then lay down new bone on chondral remnant tessues to produce 
primary bone. At this stage, remodeling starts to occur, with osteoclastic resorption followed by formation of new lamellar bone [12].

We find that zoledronate $(\mathrm{ZA})$ does not prevent bone healing. The callus under the effect of ZA showed larger callus area and a significant increase of woven bone and trabecular bone. The effect of ZA was characterized by accentuated stimulation of primary bone production and probably inhibition of remodeling, leading to retention of trabecular bone.

Our findings suggest that inhibition of bone resorption did not affect the first stages of the repair process. It is important to note also that bone remodeling is not a requirement for initial fracture repair. Although osteoclasts may contribute to endochondral ossification, these processes are not inherently dependent on osteoclasts [13].

We find not only decreased resorption as expected, but also an increased amount of new bone in the callus area in the fourth week. During remodeling phase our findings support the idea that BS can decrease bone resorption while osteoblasts continue their anabolic activity. This leads to an increased amount of woven bone by the delay in resorption or retention of primary formed bone during that phase, suggesting that BS would be not merely an anticatabolic but also an anabolic drug.

The effects of BS on fracture healing have been investigated after administration of clodronate, alendronate, etidronate, and incadronate [4-6]. Most of the previous studies did not result in significant changes during fracture repair, while others have shown slight improvement in the amount of bone formed. Peter et al. [6] reported that alendronate does not inhibit bone formation; however, the callus presented a volume two to three times larger. Li et al. [5] found that incadronate causes a decrease in resorption, but there was significant increase of bone volume only in the group treated with high doses 4 weeks later. Pamidronate-treated bones also showed greater callus area and reduced remodeling, but healing was not prevented [14].

Zoledronate is more potent than any other BS and its biological actions are only similar to those of pamidronate. It is not possible to extrapolate results of one compound to others, but all of them tend to produce greater callus without healing impairment. Amanat et al. [15] investigated use of zoledronate to improve bone repair and found that the drug increases callus bone mineral content (BMC), volume, and mechanical strength. Another study conducted by McDonald et al. [16] revealed that zoledronate causes significant retention of primary trabecular bone, leading to a larger, stronger callus with reduced inhibition of primary callus remodeling. These previous studies confirm and support our findings, but none of the previous reports studied bone repair based on histomorphometric parameters.
The anabolic effect of BS has been hypothesized in several studies [17, 18]. Some experimental studies suggest that BS may protect osteocytes and osteoblasts from apoptosis induced by glucocorticoids [19]. Inhibition of osteocyte apoptosis is mediated through the opening of connection 43 hemichannels and activation of extracellular signal-regulated kinases [18].

Several other recent studies raised the intriguing possibility that BS may enhance fracture repair and related processes [17]. In a distraction osteogenesis model, Kiely et al. [7] showed that decreased catabolism allows increased net bone accumulation in the regenerate during pamidronate or zoledronate treatment. Wedemeyer et al. [20], in a murine calvarial osteolysis model, showed increased osteoblastic activity and osteoid formation; those authors hypothesized that osteoblasts exposed to a single dose of zoledronate are able to increase their bone-forming potential.

Litle et al. [17] showed that zoledronate can increase callus volume, bone mineral content, bone, and trabecular volume in a femoral critical defect model. Finally, McDonald et al. [16] reported that zoledronate increases callus volume and leads to retention of primary trabecular bone during fracture healing.

The fact that we found an increased amount of newly formed bone does not necessarily mean that more bone has formed or that zoledronate is by any means anabolic. New formed bone might simply just prevail for a longer period if bone resorption is reduced. Regardless of the reason, bone formation during healing was not decreased in our study, and more bone was found in the callus area. This confirms that zoledronate could have an anabolic result by either stimulating or mimicking increased new bone formation.

To our knowledge this is the first study to show an impressive anabolic effect of BS during fracture healing by means of histomorphometric evaluation. This is an important finding and may give support to studies that aim to prove clinical benefits of these drugs in pathologic conditions in which bone repair may be involved such as pseudarthrosis, pathologic fracture, incorporation of bone grafts, and of course, healing of osteotomy in patients suffering from metabolic bone diseases.

Conflict of interest None.

\section{References}

1. Russell RGG (2006) Bisphosphonates: from bench to bedside. Ann N Y Acad Sci 1068:367-401

2. Russell RGG (2007) Bisphosphonates: mode of action and pharmacology. Pediatrics 119:S150-S162

3. Russell RG, Croucher PI, Rogers MJ (1999) Bisphosphonates: pharmacology, mechanisms of action and clinical uses. Osteoporosis Int 9:566-580 
4. Madsen JE, Berg-Larsen T, Kirkeby OJ, Falch JA, Nordsletten L (1998) No adverse effects of clodronate on fracyure healing in rats. Acta Orthop Scand 69:532-536

5. Li J, Mori S, Kaji Y, Kawanishi J, Akiyama T, Norimatsu H (2000) Concentration of bisphosphonate (incadronate) in callus area and its effects on fracture healing in rats. J Bone Miner Res 15:2240-2251

6. Peter CP, Cook WO, Nunamaker DM, Provost MT, Seedor JG, Rodan GA (1996) Effect of alendronate on fracture healing and bone remodeling in dogs. J Orthop Res 14:79-84

7. Kiely P, Ward K, Bellamore MC, Briody J, Cowell CT, Little DG (2007) Bisphosphonate rescue in distraction osteogenesis: a case series. J Pediatr Orthop 27(4):467-471

8. Matos MA, Araújo FP, Paixão FB (2007) The effect of zoledronate on bone remodeling during the healing process. Acta $\mathrm{Cir}$ Bras 22:115-119

9. Matos MA, Gonçalves RR, Araújo FP (2001) Experimental model for osteotomy in immature rabbit. Acta Ortop Bras 9(4):21-26

10. Matos MA, Araújo FP, Paixão FB (2008) Histomorphometric evaluation of bone healing in rabbit fibular odteotomy model without fixation. J Orthop Surg Res 29(3):4

11. Parfitt AM, Drezner MK, Glorieux FH, Kanis JA, Malluche H, Meunier PJ, Ott SM, Recker RR (1987) Bone histomorphometry. Standardization of nomeclature, symbols and units. J Bone and Miner Res 2:595-610

12. Little DG, Ramachandran M, Schindeler A (2007) The anabolic and catabolic responses in bone repair. J Bone Joint Surg Br 9(4):425-433

13. Deckers MM, Van Beek ER, Van Der Pluijim G, Wetterwald A, Van Der Wee-Pals L, Cecchini MG, Papapoulos SE, Löwik CW
(2002) Dissociation of angiogenesis and osteoclastogenesis during endochondral bone formation in neonatal mice. J Bone Miner Res 17:998-1007

14. Goodship AE, Walker PC, McNally D, Chambers T, Green JR (1994) Use of a bisphosphonate (pamidronate) to modulate fracture repair in ovine bon. Ann Oncol 5(Suppl 7):S53-S55

15. Amanat N, Mcdonald M, Godfrey C, Bilston L, Little D (2007) Optimal timing of bolus intravenous zoledronic acid in a rat fracture model. J Bone Miner Res 22(6):867-876

16. McDonald MM, Dulai SK, Godfrey C, Sztynda T, Little DG (2008) Bolus or weekly zoledronic acid administration does not delay endochondral fracture repair but weekly dosing enhances delays in hard callus remodeling. Bone 43(4):653-662

17. Little DG, McDonald M, Bransford R, Hodfrey CB, Amanat N (2005) Manipulation of the anabolic and catabolic reponses with OP-1 and zoledronic acid in a rat clinical defect model. J Bone Miner Res 20:2044-2052

18. Plotkin LI, Aguirre JI, Kousteni S, Manolagas SC, Bellido T (2005) Bisphosphonates and estrogens inhibit osteocyte apoptosis via distinct molecular mechanism downstream of extracellular signal-regulated kinase activation. J Biol Chem 280(8):73177325

19. Plotkin LI, Weinstein RS, Parfitt AM, Roberson PK, Manolagas SC, Bellido T (1999) Prevention of osteocyte and osteoblast apoptosis by bisphosphonates and calcitonin. J Clin Invest 104(10):1363-1374

20. Wedemeyer C, Von Knoch F, Pingsmann A, Hilken G, Sprecher C, Saxler G, Henschke F, Loer F, Von Knoch M (2005) Stimulation of bone formation by zoledronic acid in particle induced osteolysis. Biomaterials 17:3719-3727 